JOURNAL OF THE

CHUNGCHEONG MATHEMATICAL SOCIETY

Volume 26, No. 2, May 2013

\title{
THE ONE-SIDED QUADRANGULAR FUZZY SETS
}

\author{
Yong SiK Yun* AND BongJu LeE**
}

\begin{abstract}
We define one-sided quadrangular fuzzy sets, a left quadrangular fuzzy set and a right quadrangular fuzzy set. And then we generalize the results of addition, subtraction, multiplication, and division based on the Zadeh's extension principle for two one-sided quadrangular fuzzy sets. In addtion, we find the condition that the result of addition or subtraction for two one-sided quadrangular fuzzy sets becomes a triangular fuzzy number.
\end{abstract}

\section{Introduction}

We can already find so many generalized results of four operations based on Zadeh's extension principle for two triangular fuzzy numbers, two generalized triangular fuzzy sets, and two generalized trapezoidal fuzzy sets([1], [2]). Nevertheless, there are not the results of quadrangular fuzzy sets.

In this paper, we consider the results of operations for two quadrangular fuzzy sets, in particular for two one-sided quadrangular fuzzy sets. Thus we define one-sided quadrangular fuzzy sets and generalize the resutls of four operations for them. The four operations for two one-sided quadrangular fuzzy sets are based on the Zadeh's extension principle just like our other papers([1], [2]). The definition of the operations for two fuzzy sets, $\left(A, \mu_{A}\right)$ and $\left(B, \mu_{B}\right)$, is given in definition 2.4 once again.

Meanwhile, we define a pentagonal fuzzy number in definition 2.3 to generalize the results of four operations for two one-sided quadrangular fuzzy sets. In addtion, we look for the conditions that the result of addition or subtraction for two one-sided quadrangular fuzzy sets becomes a triangular fuzzy number, and provide some examples for them.

Received November 29, 2012; Accepted January 11, 2013.

2010 Mathematics Subject Classification: Primary 47N30.

Key words and phrases: a one-sided quadrangular fuzzy set, Zadeh's extension principle.

Correspondence should be addressed to Bongju Lee, leebj@knu.ac.kr. 


\section{Preliminaries}

We use the following definition 2.1. which is already defined([2]) to generalize the results of four operations for two fuzzy sets.

Definition 2.1. The set $A_{\alpha}=\left\{x \in X \mid \mu_{A}(x) \geq \alpha\right\}$ is said to be the $\alpha$-cut of a fuzzy set $A$.

The following definition of a triangular fuzzy number is already used in a few of papers([1], [2]).

Definition 2.2. A triangular fuzzy number is a fuzzy set $A=\left(a_{1}, a_{2}\right.$, $a_{3}$ ) having membership function

$$
\mu_{A}(x)= \begin{cases}0, & x<a_{1}, a_{3} \leq x \\ \frac{x-a_{1}}{a_{2}-a_{1}}, & a_{1} \leq x<a_{2} \\ \frac{a_{3}-x}{a_{3}-a_{2}}, & a_{2} \leq x<a_{3} .\end{cases}
$$

We define a pentagonal fuzzy number to generalize the results of this paper as follows.

Definition 2.3. A pentagonal fuzzy number is a fuzzy set $A$ having membership function

$$
\mu_{A}(x)= \begin{cases}0, & x<a_{1}, a_{5} \leq x \\ \frac{x-a_{1}}{2\left(a_{2}-a_{1}\right)}, & a_{1} \leq x<a_{2} \\ \frac{x-a_{3}}{2\left(a_{3}-a_{2}\right)}+1, & a_{2} \leq x<a_{3} \\ \frac{a_{3}-x}{2\left(a_{4}-a_{3}\right)}+1, & a_{3} \leq x<a_{4} \\ \frac{a_{5}-x}{2\left(a_{5}-a_{4}\right)}, & a_{4} \leq x<a_{5}\end{cases}
$$

where $a_{i} \in \mathbb{R}, i=1,2,3,4,5, a_{1}+a_{3} \neq 2 a_{2}$, and $a_{3}+a_{5} \neq 2 a_{4}$. This pentagonal fuzzy number is denoted by $A=\left(a_{1}, a_{2}, a_{3}, a_{4}, a_{5}\right)$.

The following definition of four operations for two fuzzy sets is based on the Zadeh's extention principle([3], [4], [5]).

Definition 2.4. The addition, subtraction, multiplication, and division of two fuzzy sets, $A$ and $B$, are defined as

1. $A(+) B: \mu_{A(+) B}(z)=\sup _{z=x+y} \min \left\{\mu_{A}(x), \mu_{B}(y)\right\}, x \in A, y \in B$

2. $A(-) B: \mu_{A(-) B}(z)=\sup _{z=x-y} \min \left\{\mu_{A}(x), \mu_{B}(y)\right\}, x \in A, y \in B$

3. $A(\cdot) B: \mu_{A(\cdot) B}(z)=\sup _{z=x y} \min \left\{\mu_{A}(x), \mu_{B}(y)\right\}, x \in A, y \in B$

4. $A(/) B: \mu_{A(/) B}(z)=\sup _{z=x / y} \min \left\{\mu_{A}(x), \mu_{B}(y)\right\}, x \in A, y \in B$. 
We define a one-sided quadrangular fuzzy set and generalize about four operations for two left(right) quadrangular fuzzy sets in the following section 3. In particular, we investigate, given a left quadrangular fuzzy set $A$, there is a left quadrangular fuzzy set $B$ such that $A(+) B$ becomes a triangular fuzzy number. Similarly, we show, given a right quadrangular fuzzy set $A$, there is a left quadrangular fuzzy set $B$ such that $A(-) B$ becomes a triangular fuzzy number.

\section{The one-sided quadrangular fuzzy sets}

Definition 3.1. A left quadrangular fuzzy set is a fuzzy set $A$ having membership function

$$
\mu_{A}(x)= \begin{cases}0, & x<a_{1}, a_{4} \leq x \\ \frac{x-a_{1}}{2\left(a_{2}-a_{1}\right)}, & a_{1} \leq x<a_{2} \\ \frac{x-a_{3}}{2\left(a_{3}-a_{2}\right)}+1, & a_{2} \leq x<a_{3} \\ \frac{a_{4}-x}{a_{4}-a_{3}}, & a_{3} \leq x<a_{4}\end{cases}
$$

where $a_{i} \in \mathbb{R}, i=1,2,3,4$ and $a_{1}+a_{3} \neq 2 a_{2}$. This left quadrangular fuzzy set is denoted by $A=\left(a_{1}, a_{2}^{*}, a_{3}, a_{4}\right)$.

REMARK 3.2. In particular, in order for a left quadrangular $A$ to be a triangular fuzzy number, it is necessary that $2 a_{2}=a_{1}+a_{3}$ should be met.

Proof. In order for $A$ to be a triangular fuzzy number, the slope of $\mu_{A}(x)$ in $\left[a_{1}, a_{2}\right]$ should be same to the slope in $\left[a_{2}, a_{3}\right]$. It means that $\frac{1}{2\left(a_{2}-a_{1}\right)}=\frac{1}{2\left(a_{3}-a_{2}\right)}$. Therefore, it is $2 a_{2}=a_{1}+a_{3}$.

Definition 3.3. A right quadrangular fuzzy set is a fuzzy set $A$ having membership function

$$
\mu_{A}(x)= \begin{cases}0, & x<a_{1}, a_{4} \leq x \\ \frac{x-a_{1}}{a_{2}-a_{1}}, & a_{1} \leq x<a_{2} \\ \frac{a_{2}-x}{2\left(a_{3}-a_{2}\right)}+1, & a_{2} \leq x<a_{3} \\ \frac{a_{4}-x}{2\left(a_{4}-a_{3}\right)}, & a_{3} \leq x<a_{4}\end{cases}
$$

where $a_{i} \in \mathbb{R}, i=1,2,3,4$ and $a_{2}+a_{4} \neq 2 a_{3}$. This right quadrangular fuzzy set is denoted by $A=\left(a_{1}, a_{2}, a_{3}^{*}, a_{4}\right)$.

REMARK 3.4. In particular, in order for a right quadrangular $A$ to be a triangular fuzzy number, it is necessary that $2 a_{3}=a_{2}+a_{4}$ should be met. 
Firstly, we generalize the results of addition, subtraction, multiplication, and division for two left quadrangular fuzzy sets in the following thm 3.5.

Theorem 3.5. For two left quadrangular fuzzy sets, $A=\left(a_{1}, a_{2}^{*}, a_{3}\right.$, $\left.a_{4}\right)$ and $B=\left(b_{1}, b_{2}^{*}, b_{3}, b_{4}\right)$, the results of four operations are as follows;

1. $A(+) B$ is a left quadrangular fuzzy set or a triangular fuzzy number.

2. $A(-) B$ is a pentagonal fuzzy number.

3. $A(\cdot) B$ is a fuzzy set on $\left(a_{1} b_{1}, a_{4} b_{4}\right)$, but doesn't need to be a left quadrangular fuzzy set or a pentagonal fuzzy number.

4. $A(/) B$ is a fuzzy set on $\left(\frac{a_{1}}{b_{4}}, \frac{a_{4}}{b_{1}}\right)$, but doesn't need to be a left quadrangular fuzzy set or a pentagonal fuzzy number.

Proof. Note that

$$
\mu_{A}(x)= \begin{cases}0, & x<a_{1}, a_{4} \leq x \\ \frac{x-a_{1}}{2\left(a_{2}-a_{1}\right)}, & a_{1} \leq x<a_{2} \\ \frac{x-a_{3}}{2\left(a_{3}-a_{2}\right)}+1, & a_{2} \leq x<a_{3} \\ \frac{a_{4}-x}{a_{4}-a_{3}}, & a_{3} \leq x<a_{4}\end{cases}
$$

and

$$
\mu_{B}(x)= \begin{cases}0, & x<b_{1}, b_{4} \leq x \\ \frac{x-b_{1}}{2\left(b_{2}-b_{1}\right)}, & b_{1} \leq x<b_{2} \\ \frac{x-b_{3}}{2\left(b_{3}-b_{2}\right)}+1, & b_{2} \leq x<b_{3} \\ \frac{b_{4}-x}{b_{4}-b_{3}}, & b_{3} \leq x<b_{4}\end{cases}
$$

Four operations are calculated by using $\alpha$-cuts. Let $A_{\alpha}=\left[a_{1}^{(\alpha)}, a_{2}^{(\alpha)}\right]$ and $B_{\alpha}=\left[b_{1}^{(\alpha)}, b_{2}^{(\alpha)}\right]$ are the $\alpha$-cuts of $A$ and $B$. We have to consider $\alpha$-cuts for two cases, $0<\alpha \leq \frac{1}{2}$ and $\frac{1}{2}<\alpha \leq 1$. First, $\alpha=\frac{a_{1}^{(\alpha)}-a_{1}}{2\left(a_{2}-a_{1}\right)}$ and $\alpha=\frac{a_{4}-a_{2}^{(\alpha)}}{2\left(a_{4}-a_{3}\right)}$ for $0<\alpha \leq \frac{1}{2}$. Thus,

$$
A_{\alpha}=\left[2 \alpha\left(a_{2}-a_{1}\right)+a_{1}, \alpha\left(a_{3}-a_{4}\right)+a_{4}\right]
$$

and

$$
B_{\alpha}=\left[2 \alpha\left(b_{2}-b_{1}\right)+b_{1}, \alpha\left(b_{3}-b_{4}\right)+b_{4}\right] \text {. }
$$

Secondly, $\alpha=\frac{a_{2}-a_{1}^{(\alpha)}}{2\left(a_{3}-a_{2}\right)}+1$ and $\alpha=\frac{a_{4}-a_{2}^{(\alpha)}}{2\left(a_{4}-a_{3}\right)}$ for $\frac{1}{2}<\alpha \leq 1$. Thus, 
and

$$
A_{\alpha}=\left[2(\alpha-1)\left(a_{3}-a_{2}\right)+a_{3}, \alpha\left(a_{3}-a_{4}\right)+a_{4}\right]
$$

$$
B_{\alpha}=\left[2(\alpha-1)\left(b_{3}-b_{2}\right)+b_{3}, \alpha\left(b_{3}-b_{4}\right)+b_{4}\right] .
$$

1. Addition : For $0<\alpha \leq \frac{1}{2}$,

$$
\begin{aligned}
A_{\alpha}(+) B_{\alpha}= & {\left[a_{1}^{(\alpha)}+b_{1}^{(\alpha)}, a_{2}^{(\alpha)}+b_{2}^{(\alpha)}\right] } \\
= & {\left[2 \alpha\left(a_{2}-a_{1}\right)+a_{1}+2 \alpha\left(b_{2}-b_{1}\right)+b_{1}\right.} \\
& \left.\quad 2(\alpha-1)\left(a_{3}-a_{2}\right)+a_{3}+2(\alpha-1)\left(b_{3}-b_{2}\right)+b_{3}\right]
\end{aligned}
$$

and for $\frac{1}{2}<\alpha \leq 1$,

$$
\begin{gathered}
A_{\alpha}(+) B_{\alpha}=\left[2(\alpha-1)\left(a_{3}-a_{2}\right)+a_{3}+2(\alpha-1)\left(b_{3}-b_{2}\right)+b_{3},\right. \\
\left.\alpha\left(a_{3}-a_{4}\right)+a_{4}+\alpha\left(b_{3}-b_{4}\right)+b_{4}\right]
\end{gathered}
$$

If $x \in\left[a_{1}+b_{1}, a_{2}+b_{2}\right]$, then $x=2 \alpha\left(a_{2}-a_{1}\right)+a_{1}+2 \alpha\left(b_{2}-b_{1}\right)+b_{1}$. Thus $\alpha=\frac{x-a_{1}-b_{1}}{2\left(a_{2}+b_{2}-a_{1}-b_{1}\right)}$. Similarly, we obtain $\alpha=\frac{x-a_{3}-b_{3}}{2\left(a_{3}+b_{3}-a_{2}-b_{2}\right)}+1$ for $x \in\left[a_{2}+b_{2}, a_{3}+b_{3}\right]$ and $\alpha=\frac{a_{4}+b_{4}-x}{a_{4}+b_{4}-a_{3}-b_{3}}$ for $x \in\left[a_{3}+b_{3}, a_{4}+b_{4}\right]$. Thus

$$
\mu_{A(+) B}(x)= \begin{cases}0, & x<a_{1}+b_{1}, a_{4}+b_{4} \leq x \\ \frac{x-a_{1}-b_{1}}{2\left(a_{2}+b_{2}-a_{1}-b_{1}\right)}, & a_{1}+b_{1} \leq x<a_{2}+b_{2} \\ \frac{x-a_{3}-b_{3}}{2\left(a_{3}+b_{3}-a_{2}-b_{2}\right)}+1, & a_{2}+b_{2} \leq x<a_{3}+b_{3} \\ \frac{a_{4}+b_{4}-x}{a_{4}+b_{4}-a_{3}-b_{3}}, & a_{3}+b_{3} \leq x<a_{4}+b_{4} .\end{cases}
$$

It generally satisfies $a_{1}+b_{1}+a_{3}+b_{3} \neq 2\left(a_{2}+b_{2}\right)$ because of $a_{1}+a_{3} \neq 2 a_{2}$ and $b_{1}+b_{3} \neq 2 b_{2}$, but it can satisfy $a_{1}+b_{1}+a_{3}+b_{3}=2\left(a_{2}+b_{2}\right)$. If $a_{1}+$ $b_{1}+a_{3}+b_{3} \neq 2\left(a_{2}+b_{2}\right)$ then $A(+) B=\left(a_{1}+b_{1},\left(a_{2}+b_{2}\right)^{*}, a_{3}+b_{3}, a_{4}+b_{4}\right)$ and if $a_{1}+b_{1}+a_{3}+b_{3}=2\left(a_{2}+b_{2}\right)$ then $A(+) B=\left(a_{1}+b_{1}, a_{3}+b_{3}, a_{4}+b_{4}\right)$. Therefore, $A(+) B$ is a left quadrangular fuzzy set or a triangular fuzzy number.

2. Subtraction : For $0<\alpha \leq \frac{1}{2}$,

$$
\begin{aligned}
A_{\alpha}(-) B_{\alpha}= & {\left[a_{1}^{(\alpha)}-b_{2}^{(\alpha)}, a_{2}^{(\alpha)}-b_{1}^{(\alpha)}\right] } \\
= & {\left[2 \alpha\left(a_{2}-a_{1}\right)+a_{1}-\alpha\left(b_{3}-b_{4}\right)-b_{4}\right.} \\
& \left.\quad \alpha\left(a_{3}-a_{4}\right)+a_{4}-2 \alpha\left(b_{2}-b_{1}\right)-b_{1}\right]
\end{aligned}
$$


and for $\frac{1}{2}<\alpha \leq 1$,

$$
\begin{array}{r}
A_{\alpha}(-) B_{\alpha}=\left[2(\alpha-1)\left(a_{3}-a_{2}\right)+a_{3}-\alpha\left(b_{3}-b_{4}\right)-b_{4},\right. \\
\left.\alpha\left(a_{3}-a_{4}\right)+a_{4}-2(\alpha-1)\left(b_{3}-b_{2}\right)-b_{3}\right] .
\end{array}
$$

If $x \in\left[a_{1}-b_{4}, a_{2}-\frac{b_{3}+b_{4}}{2}\right]$, then $x=2 \alpha\left(a_{2}-a_{1}\right)+a_{1}-\alpha\left(b_{3}-b_{4}\right)-b_{4}$ and $\alpha=\frac{x-a_{1}+b_{4}}{2 a_{2}-2 a_{1}+b_{4}-b_{3}}$. Similarly, we have $\alpha=\frac{x-a_{3}+b_{3}}{2 a_{3}-2 a_{2}+b_{4}-b_{3}}+1$ for $x \in\left[a_{2}-\frac{b_{3}+b_{4}}{2}, a_{3}-b_{3}\right], \alpha=\frac{a_{3}-b_{3}-x}{a_{4}-a_{3}+2 b_{3}-2 b_{2}}+1$ for $x \in\left[a_{3}-\right.$ $\left.b_{3}, \frac{a_{3}+a_{4}}{2}-b_{2}\right]$, and $\alpha=\frac{a_{4}-b_{1}-x}{a_{4}-a_{3}+2 b_{2}-2 b_{1}}$ for $x \in\left[\frac{a_{3}+a_{4}}{2}-b_{2}, a_{4}-b_{1}\right]$. Thus

$$
\mu_{A(-) B}(x)= \begin{cases}0, & x<a_{1}-b_{4}, a_{4}-b_{1} \leq x \\ \frac{x-a_{1}+b_{4}}{2 a_{2}-2 a_{1}+b_{4}-b_{3}}, & a_{1}-b_{4} \leq x<a_{2}-\frac{b_{3}+b_{4}}{2} \\ \frac{x-a_{3}+b_{3}}{2 a_{3}-2 a_{2}-b_{3}+b_{4}}+1, & a_{2}-\frac{b_{3}+b_{4}}{2} \leq x<a_{3}-b_{3} \\ \frac{a_{3}-b_{3}-x}{a_{4}-a_{3}+2 b_{3}-2 b_{2}}+1, & a_{3}-b_{3} \leq x<\frac{a_{3}+a_{4}}{2}-b_{2} \\ \frac{a_{4}-b_{1}-x}{a_{4}-a_{3}+2 b_{2}-2 b_{1}}, & \frac{a_{3}+a_{4}}{2}-b_{2} \leq x<a_{4}-b_{1} .\end{cases}
$$

Also, it satisfies that $2\left(a_{2}-\frac{b_{3}+b_{4}}{2}\right) \neq a_{1}-b_{4}+a_{3}-b_{3}$ and $2\left(\frac{a_{3}+a_{4}}{2}-\right.$ $\left.b_{2}\right) \neq a_{3}-b_{3}+a_{4}-b_{1}$ because of $2 a_{2} \neq a_{1}+a_{3}$ and $2 b_{2} \neq b_{1}+b_{3}$. Therefore, $A(-) B$ is a pentagonal fuzzy number and it is denoted by $A(-) B=\left(a_{1}-b_{4}, a_{2}-\frac{b_{3}+b_{4}}{2}, a_{3}-b_{3}, \frac{a_{3}+a_{4}}{2}-b_{2}, a_{4}-b_{1}\right)$.

3. Multiplication : For $0<\alpha \leq \frac{1}{2}$,

$$
\begin{aligned}
A_{\alpha}(\cdot) B_{\alpha}= & {\left[a_{1}^{(\alpha)} b_{1}^{(\alpha)}, a_{2}^{(\alpha)} b_{2}^{(\alpha)}\right] } \\
= & {\left[\left\{2 \alpha\left(a_{2}-a_{1}\right)+a_{1}\right\}\left\{2 \alpha\left(b_{2}-b_{1}\right)+b_{1}\right\},\right.} \\
& \left.\left\{\alpha\left(a_{3}-a_{4}\right)+a_{4}\right\}\left\{\alpha\left(b_{3}-b_{4}\right)+b_{4}\right\}\right]
\end{aligned}
$$

and for $\frac{1}{2}<\alpha \leq 1$,

$$
\begin{gathered}
A_{\alpha}(\cdot) B_{\alpha}=\left[\left\{2(\alpha-1)\left(a_{3}-a_{2}\right)+a_{3}\right\}\left\{2(\alpha-1)\left(b_{3}-b_{2}\right)+b_{3}\right\},\right. \\
\left.\left\{\alpha\left(a_{3}-a_{4}\right)+a_{4}\right\}\left\{\alpha\left(b_{3}-b_{4}\right)+b_{4}\right\}\right] .
\end{gathered}
$$

If $x \in\left[a_{1} b_{1}, a_{2} b_{2}\right]$, then $x=\left\{2 \alpha\left(a_{2}-a_{1}\right)+a_{1}\right\}\left\{2 \alpha\left(b_{2}-b_{1}\right)+b_{1}\right\}$. Thus $\alpha=\frac{1}{4 p q}\left(-b_{1} p-a_{1} q+\sqrt{\left(b_{1} p+a_{1} q\right)^{2}-4 p q\left(a_{1} b_{1}-x\right)}\right)$ where $p=$ $a_{2}-a_{1}$ and $q=b_{2}-b_{1}$. Similarly, we obtain $\alpha=\frac{1}{4 r s}\left(4 r s-b_{3} r-\right.$ $\left.a_{3} s+\sqrt{\left(4 r s-b_{3} r-a_{3} s\right)^{2}-4 r s\left(4 r s-2 b_{3} r-2 a_{3} s+a_{3} b_{3}-x\right)}\right)$ where $r=a_{3}-a_{2}$ and $s=b_{3}-b_{2}$ for $x \in\left[a_{2} b_{2}, a_{3} b_{3}\right]$ and $\alpha=\frac{1}{4 t u}\left(-b_{4} t-\right.$ 
$\left.a_{4} u-\sqrt{\left(b_{4} t+a_{4} u\right)^{2}-4 t u\left(a_{4} b_{4}-x\right)}\right)$ where $t=a_{3}-a_{4}$ and $s=b_{3}-b_{4}$ for $x \in\left[a_{3} b_{3}, a_{4} b_{4}\right]$. Therefore, the membership function $\mu_{A(\cdot) B}(x)$ of $A(\cdot) B$ is

$$
\left\{\begin{array}{cr}
0, & x<a_{1} b_{1}, \quad a_{4} b_{4} \leq x \\
\left.\frac{1}{4 p q}\left(-b_{1} p-a_{1} q\right)+\sqrt{\left(b_{1} p+a_{1} q\right)^{2}-4 p q\left(a_{1} b_{1}-x\right)}\right), & a_{1} b_{1} \leq x<a_{2} b_{2} \\
\frac{1}{4 r s}\left(\sqrt{\left(4 r s-b_{3} r-a_{3} s\right)^{2}-4 r s\left(4 r s-2 b_{3} r-2 a_{3} s+a_{3} b_{3}-x\right)}\right. & a_{2} b_{3} \leq x<a_{3} b_{3} \\
\left.+4 r s-b_{3} r-a_{3} s\right), & a_{3} b_{3} \leq x<a_{4} b_{4}
\end{array}\right.
$$

where $p=a_{2}-a_{1}, q=b_{2}-b_{1}, r=a_{3}-a_{2}, s=b_{3}-b_{2}, t=a_{3}-a_{4}$, and $s=b_{3}-b_{4}$. Hence $A(\cdot) B$ is a fuzzy set on $\left(a_{1} b_{1}, a_{4} b_{4}\right)$, but doesn't need to be a left quadrangular fuzzy set or a pentagonal fuzzy number.

4. Division : For $0<\alpha \leq \frac{1}{2}$,

$$
\begin{aligned}
A_{\alpha}(/) B_{\alpha} & =\left[a_{1}^{(\alpha)} / b_{2}^{(\alpha)}, a_{2}^{(\alpha)} / b_{1}^{(\alpha)}\right] \\
& =\left[\frac{2 \alpha\left(a_{2}-a_{1}\right)+a_{1}}{\alpha\left(b_{3}-b_{4}\right)+b_{4}}, \frac{\alpha\left(a_{3}-a_{4}\right)+a_{4}}{2 \alpha\left(b_{2}-b_{1}\right)+b_{1}}\right]
\end{aligned}
$$

and for $\frac{1}{2}<\alpha \leq 1$,

$$
A_{\alpha}(/) B_{\alpha}=\left[\frac{2(\alpha-1)\left(a_{3}-a_{2}\right)+a_{3}}{\alpha\left(b_{3}-b_{4}\right)+b_{4}}, \frac{\alpha\left(a_{3}-a_{4}\right)+a_{4}}{2(\alpha-1)\left(b_{3}-b_{2}\right)+b_{3}}\right] .
$$

If $x \in\left[a_{1} / b_{4}, a_{2} / \frac{b_{3}+b_{4}}{2}\right]$, then $x=\frac{2 \alpha\left(a_{2}-a_{1}\right)+a_{1}}{\alpha\left(b_{3}-b_{4}\right)+b_{4}}$. Thus

We also have

$$
\alpha=\frac{b_{4} x+a_{1}}{\left(b_{3}-b_{4}\right) x-2\left(a_{2}-a_{1}\right)} .
$$

$$
\begin{gathered}
\alpha=\frac{b_{4} x+2 a_{2}-a_{3}}{\left(b_{3}-b_{4}\right) x-2\left(a_{3}-a_{2}\right)} \quad \text { for } \quad x \in\left[a_{2} / \frac{b_{3}+b_{4}}{2}, a_{3} / b_{3}\right], \\
\alpha=\frac{\left(3 b_{3}-b_{2}\right) x+a_{4}}{2\left(b_{3}-b_{2}\right) x+a_{4}-a_{3}} \quad \text { for } \quad x \in\left[a_{3} / b_{3}, \frac{a_{3}+a_{4}}{2} / b_{2}\right],
\end{gathered}
$$

and

$$
\alpha=\frac{b_{1} x+a_{4}}{2\left(b_{2}-b_{1}\right) x+a_{4}-a_{3}} \quad \text { for } \quad x \in\left[\frac{a_{3}+a_{4}}{2} / b_{2}, a_{4} / b_{1}\right]
$$


Therefore

$$
\mu_{A(/) B}(x)= \begin{cases}0, & x<a_{1} / b_{4}, a_{4} / b_{1} \leq x \\ \frac{b_{4} x+a_{1}}{\left(b_{3}-b_{4}\right) x-2\left(a_{2}-a_{1}\right)}, & a_{1} / b_{4} \leq x<a_{2} / \frac{b_{3}+b_{4}}{2} \\ \frac{b_{4} x+2 a_{2}-a_{3}}{\left(b_{3}-b_{4}\right) x-2\left(a_{3}-a_{2}\right)}, & a_{2} / \frac{b_{3}+b_{4}}{2} \leq x<a_{3} / b_{3} \\ \frac{\left(3 b_{3}-b_{2}\right) x+a_{4}}{2\left(b_{3}-b_{2}\right) x+a_{4}-a_{3}}, & a_{3} / b_{3} \leq x<\frac{a_{3}+a_{4}}{2} / b_{2} \\ \frac{b_{1} x+a_{4}}{2\left(b_{2}-b_{1}\right) x+a_{4}-a_{3}}, & \frac{a_{3}+a_{4}}{2} / b_{2} \leq x<a_{4} / b_{1} .\end{cases}
$$

Hence $A(/) B$ is a fuzzy set on $\left(\frac{a_{1}}{b_{4}}, \frac{a_{4}}{b_{1}}\right)$, but doesn't need to be a left quadrangular fuzzy set or a pentagonal fuzzy number.

Remark 3.6. Given a left quadrangular fuzzy set $A$, there is a left quadrangular fuzzy set $B$ such that $A(+) B$ becomes a triangular fuzzy number.

Proof. Let $A=\left(a_{1}, a_{2}^{*}, a_{3}, a_{4}\right)$. We seek a function based on the left quadrangular fuzzy set $B=\left(b_{1}, b_{2}^{*}, b_{3}, b_{4}\right)$. Since $b_{1}$ and $b_{4}$ are arbitrary we can randomly take them. Then $b_{2}$ and $b_{3}$ should satisfy the following relation

$$
\frac{1}{2\left(a_{2}+b_{2}-a_{1}-b_{1}\right)}=\frac{1}{2\left(a_{3}+b_{3}-a_{2}-b_{2}\right)}
$$

Therefore, we have $b_{3}=2 a_{2}+2 b_{2}-a_{1}-a_{3}-b_{1}$.

ExAmple 3.7. For $A=\left(1,2^{*}, 4,5\right)$, we have $B=\left(3,6^{*}, 8,9\right)$ that $A(+) B$ becomes a triangular fuzzy number. Since $b_{3}=-a_{1}-b_{1}+2 a_{2}+$ $2 b_{2}-a_{3}, A(+) B=(4,12,14)$ and

$$
\mu_{A(+) B}(x)= \begin{cases}0, & x<4,14 \leq x \\ \frac{x-4}{8}, & 4 \leq x<12 \\ \frac{14-x}{2}, & 12 \leq x<14\end{cases}
$$

Subsequently, we generalize the results of four operations for two right quadrangular fuzzy sets in the following theorem 3.8. For that, let $A=\left(a_{1}, a_{2}, a_{3}^{*}, a_{4}\right)$ and $B=\left(b_{1}, b_{2}, b_{3}^{*}, b_{4}\right)$, where $a_{i}, b_{i} \in \mathbb{R}, i=1,2,3,4$, $a_{2}+a_{4} \neq 2 a_{3}$, and $b_{2}+b_{4} \neq 2 b_{3}$.

TheOrem 3.8. For two right quadrangular fuzzy sets, $A=\left(a_{1}, a_{2}, a_{3}^{*}\right.$, $\left.a_{4}\right)$ and $B=\left(b_{1}, b_{2}, b_{3}^{*}, b_{4}\right)$, the results of four operations are as follows;

1. $A(+) B$ is a right quadrangular fuzzy set or a triangular fuzzy number.

2. $A(-) B$ is a pentagonal fuzzy number.

3. $A(\cdot) B$ is a fuzzy set on $\left(a_{1} b_{1}, a_{4} b_{4}\right)$, but doesn't need to be a right quadrangular fuzzy set or a pentagonal fuzzy number. 
4. $A(/) B$ is a fuzzy set on $\left(\frac{a_{1}}{b_{4}}, \frac{a_{4}}{b_{1}}\right)$, but doesn't need to be a right quadrangular fuzzy set or a pentagonal fuzzy number.

Proof. The proof is similar with Theorem 3.5.

REMARK 3.9. Given a right quadrangular fuzzy set $A$, there is a right quadrangular fuzzy set $B$ such that $A(+) B$ becomes a triangular fuzzy number.

Proof. The proof is similar with Remark 3.6.

We generalize about addition and subtraction for a left quadrangular fuzzy set and a right quadrangular fuzzy set. For that, let $A=$ $\left(a_{1}, a_{2}^{*}, a_{3}, a_{4}\right)$ and $B=\left(b_{1}, b_{2}, b_{3}^{*}, b_{4}\right)$, where $a_{i}, b_{i} \in \mathbb{R}, i=1,2,3,4$, $a_{1}+a_{3} \neq 2 a_{2}$, and $b_{2}+b_{4} \neq 2 b_{3}$. Meanwhile it's better to consider $B(-) A$ because $A(-) B \neq B(-) A$.

Theorem 3.10. For a left quadrangular fuzzy set $A$ and a right quadrangular fuzzy set $B$, the results of $A(+) B, B(+) A, A(-) B$, and $B(-) A$ are as follows;

1. $A(+) B$ and $B(+) A$ are the pentagonal fuzzy numbers.

2. $A(-) B$ is a left quadrangular fuzzy set or a triangular fuzzy number.

3. $B(-) A$ is a right quadrangular fuzzy set or a triangular fuzzy number.

Proof. We calculate addition and subtration using $\alpha$-cuts as in the preceding. Let $A_{\alpha}=\left[a_{1}^{(\alpha)}, a_{2}^{(\alpha)}\right]$ and $B_{\alpha}=\left[b_{1}^{(\alpha)}, b_{2}^{(\alpha)}\right]$ are the $\alpha$-cuts of $A$ and $B$. We use $A_{\alpha}$ and $B_{\alpha}$ in Theorem 3.5 for two cases. First, $\alpha=\frac{b_{1}^{(\alpha)}-b_{1}}{b_{2}-b_{1}}$ and $\alpha=\frac{b_{4}-b_{2}^{(\alpha)}}{2\left(b_{4}-b_{3}\right)}$ for $0<\alpha \leq \frac{1}{2}$. Secondly, $\alpha=\frac{b_{1}^{(\alpha)-b_{1}}}{b_{2}-b_{1}}$ and $\alpha=\frac{b_{2}-b_{2}^{(\alpha)}}{2\left(b_{3}-b_{2}\right)}+1$ for $\frac{1}{2}<\alpha \leq 1$. Thus, for $0<\alpha \leq \frac{1}{2}$,

$$
\begin{gathered}
A_{\alpha}=\left[2 \alpha\left(a_{2}-a_{1}\right)+a_{1}, \alpha\left(a_{3}-a_{4}\right)+a_{4}\right], \\
B_{\alpha}=\left[\alpha\left(b_{2}-b_{1}\right)+b_{1}, 2 \alpha\left(b_{4}-b_{3}\right)+b_{4}\right]
\end{gathered}
$$

and for $\frac{1}{2}<\alpha \leq 1$,

$$
\begin{aligned}
& A_{\alpha}=\left[2(\alpha-1)\left(a_{3}-a_{2}\right)+a_{3}, \alpha\left(a_{3}-a_{4}\right)+a_{4}\right], \\
& B_{\alpha}=\left[\alpha\left(b_{2}-b_{1}\right)+b_{1}, 2(\alpha-1)\left(b_{3}-b_{2}\right)+b_{2}\right] .
\end{aligned}
$$


1. $A(+) B$ and $B(+) A$ : For $0<\alpha \leq \frac{1}{2}$,

$$
\begin{aligned}
& A_{\alpha}(+) B_{\alpha}=\left[2 \alpha\left(a_{2}-a_{1}\right)+a_{1}+\alpha\left(b_{2}-b_{1}\right)+b_{1},\right. \\
& \left.\alpha\left(a_{3}-a_{4}\right)+a_{4}-2 \alpha\left(b_{4}-b_{3}\right)+b_{4}\right]
\end{aligned}
$$

and for $\frac{1}{2}<\alpha \leq 1$,

$$
\begin{array}{r}
A_{\alpha}(+) B_{\alpha}=\left[2(\alpha-1)\left(a_{3}-a_{2}\right)+a_{3}+\alpha\left(b_{2}-b_{1}\right)+b_{1},\right. \\
\left.\alpha\left(a_{3}-a_{4}\right)+a_{4}-2(\alpha-1)\left(b_{3}-b_{2}\right)+b_{2}\right] .
\end{array}
$$

If $x \in\left[a_{1}+b_{1}, a_{2}+\frac{b_{1}+b_{2}}{2}\right]$, then $x=2 \alpha\left(a_{2}-a_{1}\right)+a_{1}+\alpha\left(b_{2}-b_{1}\right)+b_{1}$. Thus $\alpha=\frac{x-a_{1}-b_{1}}{2 a_{2}-2 a_{1}+b_{2}-b_{1}}$. Similarly, we have $\alpha=\frac{x-a_{3}-b_{2}}{2 a_{3}-2 a_{2}+b_{2}-b_{1}}+1$ for $x \in\left[a_{2}+\frac{b_{1}+b_{2}}{2}, a_{3}+b_{2}\right], \alpha=\frac{a_{3}+b_{2}-x}{a_{4}-a_{3}+2 b_{3}-2 b_{2}}+1$ for $x \in\left[a_{3}+\right.$ $\left.b_{2}, \frac{a_{3}+a_{4}}{2}+b_{3}\right]$, and $\alpha=\frac{a_{4}+b_{4}-x}{a_{4}-a_{3}+2 b_{4}-2 b_{3}}$ for $x \in\left[\frac{a_{3}+a_{4}}{2}+b_{3}, a_{4}+b_{4}\right]$. Thus

$$
\mu_{A(+) B}(x)= \begin{cases}0, & x<a_{1}+b_{1}, a_{4}+b_{4} \leq x \\ \frac{x-a_{1}-b_{1}}{2 a_{2}-2 a_{1}+b_{2}-b_{1}}, & a_{1}+b_{1} \leq x<a_{2}+\frac{x-b_{1}+b_{2}}{2} \\ \frac{x-a_{3}-b_{2}}{2 a_{3}-2 a_{2}+b_{2}-b_{1}}+1, & a_{2}+\frac{b_{1}+b_{2}}{2} \leq x<a_{3}+b_{2} \\ \frac{a_{3}+b_{2}-x}{a_{4}-a_{3}+2 b_{3}-2 b_{2}}+1, & a_{3}+b_{2} \leq x<\frac{a_{3}+a_{4}}{2}+b_{3} \\ \frac{a_{4}+b_{4}-x}{a_{4}-a_{3}+2 b_{4}-2 b_{3}}, & \frac{a_{3}+a_{4}}{2}+b_{3} \leq x<a_{4}+b_{4} .\end{cases}
$$

Also, it satisfies that $2\left(a_{2}+\frac{b_{1}+b_{2}}{2}\right) \neq a_{1}+b_{1}+a_{3}+b_{2}$ and $2\left(\frac{a_{3}+a_{4}}{2}+\right.$ $\left.b_{3}\right) \neq a_{3}+b_{2}+a_{4}+b_{4}$ because of $2 a_{2} \neq a_{1}+a_{3}$ and $2 b_{3} \neq b_{2}+$ $b_{4}$. Therefore, $A(+) B$ is a pentagonal fuzzy number and $A(+) B=$ $\left(a_{1}+b_{1}, a_{2}+\frac{b_{1}+b_{2}}{2}, a_{3}+b_{2}, \frac{a_{3}+a_{4}}{2}+b_{3}, a_{4}+b_{4}\right)$. Similiarly, $B(+) A$ is a pentagonal fuzzy number and it is denoted by $B(+) A=\left(b_{1}+a_{1}, b_{2}+\right.$ $\left.\frac{a_{1}+a_{2}}{2}, b_{3}+a_{2}, \frac{b_{3}+b_{4}}{2}+a_{3}, b_{4}+a_{4}\right)$.

2. $A(-) B$ : For $0<\alpha \leq \frac{1}{2}$,

$$
\begin{array}{r}
A_{\alpha}(-) B_{\alpha}=\left[2 \alpha\left(a_{2}-a_{1}\right)+a_{1}-2 \alpha\left(b_{4}-b_{3}\right)-b_{4},\right. \\
\left.\alpha\left(a_{3}-a_{4}\right)+a_{4}-\alpha\left(b_{2}-b_{1}\right)-b_{1}\right]
\end{array}
$$

and for $\frac{1}{2}<\alpha \leq 1$,

$$
\begin{gathered}
A_{\alpha}(-) B_{\alpha}=\left[2(\alpha-1)\left(a_{3}-a_{2}\right)+a_{3}-2(\alpha-1)\left(b_{3}-b_{2}\right)-b_{2},\right. \\
\left.\alpha\left(a_{3}-a_{4}\right)+a_{4}-\alpha\left(b_{2}-b_{1}\right)-b_{1}\right] .
\end{gathered}
$$


We have $\alpha=\frac{x-a_{1}+b_{4}}{2\left(a_{2}-a_{1}+b_{4}-b_{3}\right)}$ for $x \in\left[a_{1}-b_{4}, a_{2}-b_{3}\right]$,

$$
\begin{gathered}
\alpha=\frac{x-a_{3}+b_{2}}{2\left(a_{3}-a_{2}+b_{3}-b_{2}\right)}+1 \text { for } x \in\left[a_{2}-b_{3}, a_{3}-b_{2}\right], \text { and } \\
\alpha=\frac{a_{4}-b_{1}-x}{a_{4}-a_{3}+b_{2}-b_{1}} \text { for } x \in\left[a_{3}-b_{2}, a_{4}-b_{1}\right] . \text { Thus } \\
\mu_{A(-) B}(x)= \begin{cases}0, & x<a_{1}-b_{4}, a_{4}-b_{1} \leq x \\
\frac{x-a_{1}+b_{4}}{2\left(a_{2}-a_{1}+b_{4}-b_{3}\right)}, & a_{1}-b_{4} \leq x<a_{2}-b_{3} \\
\frac{x-a_{3}+b_{2}}{2\left(a_{3}-a_{2}+b_{3}-b_{2}\right)}+1, & a_{2}-b_{3} \leq x<a_{3}-b_{2} \\
\frac{a_{4}-b_{1}-x}{a_{4}-a_{3}+b_{2}-b_{1}}, & a_{3}-b_{2} \leq x<a_{4}-b_{1} .\end{cases}
\end{gathered}
$$

It generally satisfies $a_{1}-b_{4}+a_{3}-b_{2} \neq 2\left(a_{2}-b_{3}\right)$ because of $a_{1}+a_{3} \neq 2 a_{2}$ and $b_{2}+b_{4} \neq 2 b_{3}$, but it can satisfy $a_{1}-b_{4}+a_{3}-b_{2}=2\left(a_{2}-b_{3}\right)$. Hence, if $a_{1}-b_{4}+a_{3}-b_{2} \neq 2\left(a_{2}-b_{3}\right)$ then $A(-) B=\left(a_{1}-b_{4},\left(a_{2}-\right.\right.$ $\left.\left.b_{3}\right)^{*}, a_{3}-b_{2}, a_{4}-b_{1}\right)$ and if $a_{1}-b_{4}+a_{3}-b_{2}=2\left(a_{2}-b_{3}\right)$ then $A(-) B=$ $\left(a_{1}-b_{4}, a_{3}-b_{2}, a_{4}-b_{1}\right)$. Therefore, $A(-) B$ is a left quadrangular fuzzy set or a triangular fuzzy number.

3. $B(-) A$ : The proof is similar with the proof of $A(-) B$.

REMARK 3.11. Given a left quadrangular fuzzy set $A$, there is a right quadrangular fuzzy set $B$ such that $A(-) B$ becomes a triangular fuzzy number.

Proof. Let $A=\left(a_{1}, a_{2}^{*}, a_{3}, a_{4}\right)$. We seek a function based on the set $B=\left(b_{1}, b_{2}, b_{3}^{*}, b_{4}\right)$. Since $b_{1}$ and $b_{4}$ are arbitrary we can randomly take them. Then $b_{2}$ and $b_{3}$ should satisfy the following relation

$$
\frac{1}{2\left(a_{2}-a_{1}+b_{4}-b_{3}\right)}=\frac{1}{2\left(a_{3}-a_{2}+b_{3}-b_{2}\right)} .
$$

Therefore, we have $b_{2}=2 b_{3}-2 a_{2}-b_{4}+a_{1}+a_{3}$.

ExAmple 3.12. For $A=\left(1,2^{*}, 4,5\right)$, we have $B=\left(3,6,7^{*}, 9\right)$ that $A(-) B$ becomes a triangular fuzzy number. Since $b_{2}=2 b_{3}-2 a_{2}-b_{4}+$ $a_{1}+a_{3}, A(-) B=(-8,-2,2)$.

REMARK 3.13. Given a right quadrangular fuzzy number $B$, there is a left quadrangular fuzzy number $A$ such that $B(-) A$ becomes a triangular fuzzy number.

Proof. The proof is similar with Remark 3.11.

EXAmple 3.14. For $B=\left(2,4,5^{*}, 8\right)$, we have $A=\left(2,3^{*}, 6,8\right)$ that $B(-) A=(-6,-2,6)$ becomes a triangular fuzzy number. 


\section{References}

[1] B. J. Lee and Y. S. Yun, The generalized trapezoidal fuzzy sets, Journal of the Chungcheong Mathematical Society. 24 (2011), no. 2, 253-266.

[2] Y. S. Yun, S. U. Ryu, and J. W. Park, The generalized triangular fuzzy sets, Journal of the Chungcheong Mathematical Society 22 (2009), no. 2, 161-170.

[3] L. A. Zadeh, The concept of a linguistic variable and its application to approximate reasoning, I Information Sciences 8 (1975), 199-249.

[4] L. A. Zadeh, The concept of a linguistic variable and its application to approximate reasoning, II Information Sciences 8 (1975), 301-357.

[5] L. A. Zadeh, The concept of a linguistic variable and its application to approximate reasoning, III Information Sciences 9 (1975), 43-80.

Department of Mathematics and Research Institute for Basic Sciences Jeju National University

Jeju 690-756, Republic of Korea

E-mail: yunys@jejunu.ac.kr

$* *$

Department of Mathematics Education

Kyungpook National University

Daegu 702-701, Republic of Korea

E-mail: leebj@knu.ac.kr 